\title{
Observation of near-field coupling in metal nanoparticle chains using far-field polarization spectroscopy
}

\author{
Stefan A. Maier, Mark L. Brongersma, Pieter G. Kik, and Harry A. Atwater* \\ Thomas J. Watson Laboratory of Applied Physics, California Institute of Technology, Pasadena, California 91125
}

(Received 12 March 2002; published 13 May 2002)

\begin{abstract}
Far-field polarization spectroscopy on chains of Au nanoparticles reveals the existence of longitudinal $(L)$ and transverse $(T)$ plasmon-polariton modes. The experimental results provide support for the validity of a recently published dipole model for electromagnetic energy transfer below the diffraction limit along chains of closely spaced metal nanoparticles. The key parameters that govern the energy transport are determined for various interparticle spacings using measurements of the resonance frequencies of $L$ and $T$ modes, yielding a bandwidth of $1.4 \times 10^{14} \mathrm{rad} / \mathrm{s}$ and a maximum group velocity of $v_{g}=4.0 \times 10^{6} \mathrm{~m} / \mathrm{s}$ for a $75 \mathrm{~nm}$-spacing.
\end{abstract}

DOI: 10.1103/PhysRevB.65.193408

PACS number(s): 78.67.Bf, 42.70.Qs, 42.82.Et, 71.45.Gm

In recent years, the interaction of light with metal nanoparticles has been the focus of intensive research. ${ }^{1,2}$ From work on single noble-metal nanoparticles, it is well established that light at the surface plasmon resonance frequency interacts strongly with metal particles and excites a collective electron motion, or plasmon. ${ }^{3}$ These resonance frequencies are typically in the visible or infrared part of the spectrum. ${ }^{1}$ For particles with a diameter much smaller than the wavelength $\lambda$ of the exciting light, plasmon excitations produce an oscillating dipole field.

Whereas until recently most work focused on large numbers of particles in disordered arrays, advances in particle synthesis and fabrication techniques allow for studies of ordered arrays of noble-metal particles. Since each excited metal nanoparticle with a diameter much smaller than the wavelength of the exciting light acts as an electric dipole, two types of electromagnetic interactions between particles can be distinguished: near-field coupling and far-field dipolar interaction. For particle spacings $d$ larger than the wavelength of light, far-field dipolar interactions with a $d^{-1}$ dependence dominate. Indeed, using broad beam illumination of regular two-dimensional gratings of Au nanoparticles with grating constants of several $100 \mathrm{~nm}$, it was shown that the far-field dipolar interactions lead to changes in the position and the width of the dipole resonance. ${ }^{4-6}$ Most work has focused on these far-field interactions between metal nanoparticles and their possible applications for the fabrication of optoelectronic materials, but relatively little is known about the nature and properties of near-field interactions of closely spaced metal nanoparticles. However, recently it was suggested that chains of closely spaced noble-metal nanoparticles could be used as linear waveguides for electromagnetic energy below the diffraction limit. ${ }^{7}$ Analysis of Au nanoparticle chains using near-field optical microscopy has indeed shown signs of collective behavior of such chains. ${ }^{8}$ It has also been shown that straight solid noble-metal nanowires can allow for electromagnetic energy transport over micrometer distances. ${ }^{9}$

In a number of recent publications, we presented experiments on macroscopic microwave analog systems ${ }^{10,11}$ and theoretical models for metal nanoparticle chain waveguides. $^{12,13}$ The results suggest that chains of closely spaced $(d \ll \lambda)$ noble-metal nanoparticles could indeed be used to guide electromagnetic (EM) energy below the diffraction limit via coupled plasmon modes. ${ }^{12,13}$ Furthermore, we determined the dispersion relation of the guided modes in these plasmon waveguides using a point-dipole model, and obtained group velocities for the energy transport on the order of $0.1 c$ in air, suggesting that information transport is possible. We also showed that EM energy can be guided around sharp corners and tee structures, and an all-optical switch based on interference operating below the diffraction limit was proposed. ${ }^{12}$

In this paper, we report on the fabrication of plasmon waveguides consisting of closely spaced $\mathrm{Au}$ nanoparticles made by electron-beam lithography and analyze their optical properties using far-field polarization spectroscopy. These measurements of the collective excitation mode of plasmon waveguides, together with our point-dipole model, ${ }^{12}$ fully determine the dispersion relation describing the propagation of all possible modes supported by the waveguide. Our results for the key parameters that govern the energy transport are in good agreement with our previous modeling. ${ }^{12,13}$

We fabricated plasmon waveguides by electron-beam lithography consisting of 80 closely spaced Au nanoparticles with a diameter of $50 \mathrm{~nm}$ and center-to-center spacings of 75,100 , and $125 \mathrm{~nm}$ respectively. Electron-beam lithography provides excellent size and distance control of the nanoparticles constituting the waveguides. In order to minimize charging effects during fabrication, the structures were fabricated on a quartz glass slide covered with a thin indiumtin-oxide layer. The layer thickness was chosen to be less than $10 \mathrm{~nm}$ to suppress the guiding of EM energy at the frequencies of interest. ${ }^{6}$ In order to allow for characterization with a high signal-to-noise ratio of these plasmon waveguides using far-field spectroscopy, we fabricated large area arrays in a $100 \times 100-\mu \mathrm{m}^{2}$ grid with a grating constant of $1.0 \mu \mathrm{m}$. Crosstalk between adjacent structures is expected to have a negligible effect on the dispersion relation since it takes place via far-field scattering of the individual nanoparticles with a distance dependence of $d^{-1}$, whereas near-field interactions of adjacent particles in each waveguide show a $d^{-3}$ dependence and dominate at small distances. ${ }^{12}$ Moreover, the grating constant of $1.0 \mu \mathrm{m}$ does not coincide with a grating order transition of increased far-field scattering, ${ }^{14}$ 


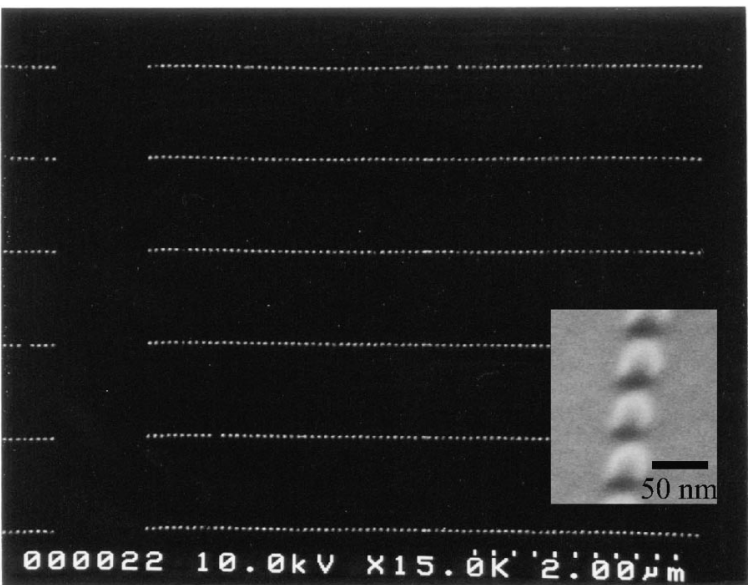

FIG. 1. Scanning electron micrograph of plasmon waveguides consisting of $\mathrm{Au}$ nanoparticles taken at a normal incidence of the electron beam. The inset shows a micrograph where the sample stage was tilted by $70^{\circ}$ to visualize the almost spherical shape of the nanoparticles.

which further minimizes the far-field dipolar interactions. Thus results from far-field spectroscopy on these arrays reflect the properties of individual plasmon waveguides. Our experimental setup consisted of a white halogen light source and a computer-operated microspectrometer attached to an inverted optical microscope, which allowed for an easy localization of the plasmon waveguide structures.

Figure 1 shows a scanning electron micrograph of several plasmon waveguides resolving individual particles. The image was taken at normal incidence of the electron beam and confirmed a diameter of $50 \mathrm{~nm}(R=25 \mathrm{~nm})$ and a center-tocenter spacing $d$ of $75 \mathrm{~nm}(3 R)$ of adjacent particles. Also, the grating constant of $1 \mu \mathrm{m}$ between neighboring structures was confirmed. Using atomic force microscopy, the height of the nanoparticles was determined to be $50 \mathrm{~nm}$. The inset of Fig. 1 shows a scanning electron micrograph of individual nanoparticles of the waveguide where the sample stage was tilted by $70^{\circ}$ in order to allow for a determination of the shape and aspect ratio of individual particles. We attribute the almost spherical shape of individual particles to roomtemperature annealing processes after the metallization and liftoff. The in-plane symmetry of the particles was confirmed by polarization spectroscopy on a regular grating of individual particles with a grating constant of $250 \mathrm{~nm}$, showing only a small inherent single particle optical anisotropy (peak shift $2 \times 10^{13} \mathrm{rad} / \mathrm{s}$ between longitudinal and the transverse modes).

Figure 2 shows a far-field extinction spectrum of plasmon waveguides with a 75-nm interparticle distance taken at normal incidence illumination with a spotsize of $100-\mu \mathrm{m}$ diameter for a polarization either in the direction of the waveguide axis (longitudinal polarization $L$ ) or perpendicular to it (transverse polarization T). Also shown is the extinction spectrum for illumination with unpolarized light $(U)$. The extinction spectra show a dipolar plasmon peak for longitudinal and transverse illumination at angular frequencies of $\omega_{\mathrm{LO}}=3.12 \times 10^{15} \mathrm{rad} / \mathrm{s}$ and $\omega_{\mathrm{TO}}=3.22 \times 10^{15} \mathrm{rad} / \mathrm{s}$, respectively, corresponding to wavelengths of maximal extinction

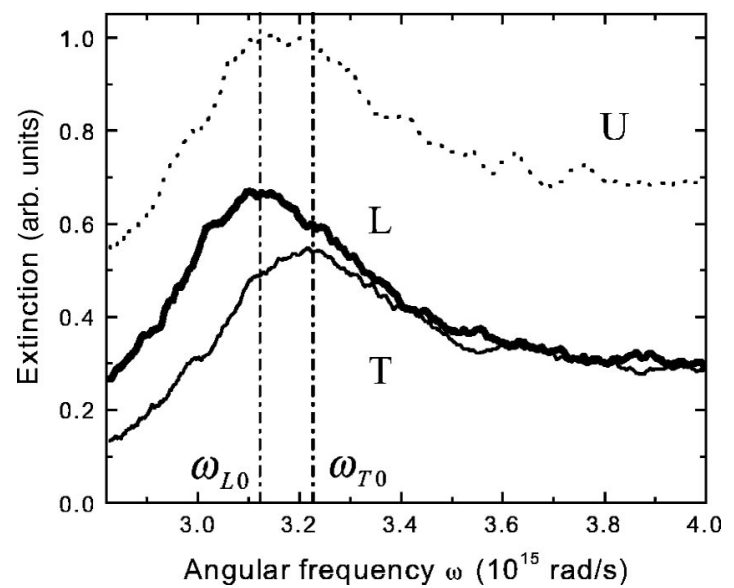

FIG. 2. Far-field extinction spectrum of plasmon waveguides for both the longitudinal (thick line $L$ ) and the transverse (thin line $T$ ) mode of collective excitation. The peak positions for the $L$ and $T$ modes (marked with straight dashed lines) are located at different frequencies due to optical near-field interactions. Also shown is the extinction spectrum for unpolarized light (dotted line $U$ ).

of 604 and $585 \mathrm{~nm}$. The extinction peak for unpolarized light lies between those two frequencies. The position of the dipole plasmon resonance is influenced both by the substrate and interparticle interactions, ${ }^{1}$ and this accounts for a shift of the resonance compared to single $\mathrm{Au}$ nanoparticles in air, which lies at $3.92 \times 10^{15} \mathrm{rad} / \mathrm{s} .{ }^{1}$ However, the polarization dependence of the peak positions indicates that near-field optical interactions are present between the nanoparticles constituting the waveguide, which is consistent with theoretical work on electromagnetic interactions between closely spaced aggregates and chains of metal nanoparticles. ${ }^{15,16}$

The polarization dependence of the plasmon resonance of the plasmon waveguides can be understood from a model that treats the Au nanoparticles as point dipoles. ${ }^{12,13}$ The inset of Fig. 3 shows the dispersion relation (frequency $\omega$ ver-

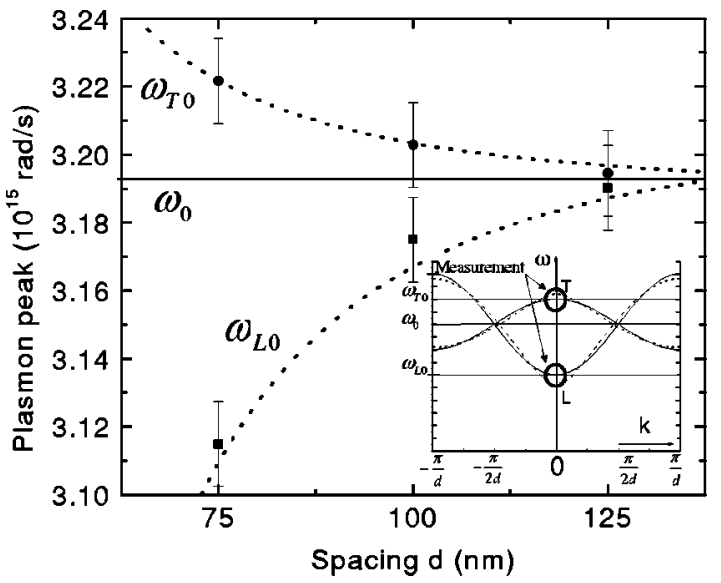

FIG. 3. Dependence of the plasmon peak position on the interparticle spacing $d$ for both the longitudinal and transverse excitation of the collective mode. The dotted line shows the $1 / d^{3}$ dependence predicted by a point dipole interaction model. The inset shows the dispersion relation of plasmon waveguides calculated using this point dipole model (Ref. 12). 
sus wave vector $\mathbf{k}$ ) of guided modes in plasmon waveguides consisting of an infinite linear array of point dipoles spaced a distance $d \ll \lambda$ apart, the calculation of which is outlined in Ref. 12. The solid lines show the dispersion relation assuming nearest-neighbor coupling only, and the dotted lines take interactions between five nearest neighbors into account. The group velocity of the energy transport is given by the slope of the dispersion relation and has a maximum at a resonance frequency $\omega_{0}$ of the waveguide. In Ref. 12 it was shown that $\omega_{0}$ corresponds to the surface plasmon resonance frequency of a single particle, and that particle chains can be excited most efficiently at this frequency using local excitation of single particles. In our experimental configuration all particles are excited in phase, corresponding to $\mathbf{k}=0$. In this case the group velocity is zero for both the longitudinal and transverse branches, and no transport of energy takes place. These collective modes occur at frequencies $\omega_{\mathrm{LO}}$ for longitudinal polarization and at $\omega_{\mathrm{TO}}$ for transverse polarization. Both are shifted with respect to the frequency $\omega_{0}$ : The longitudinal mode is shifted to lower frequencies, and the transverse mode is shifted to higher frequencies, as seen in Fig. 2. The ratio of the respective shifts is predicted to be $2: 1{ }^{12}$ The extinction maxima in the far-field extinction spectra shown in Fig. 2 directly yield values for $\omega_{\mathrm{LO}}$ and $\omega_{\mathrm{TO}}$.

We fabricated plasmon waveguides with interparticle spacings of $75 \mathrm{~nm}(3 R), 100 \mathrm{~nm}(4 R)$, and $125 \mathrm{~nm}(5 R)$ to study the influence of the interaction strength on the frequency difference $\Delta \omega=\omega_{\mathrm{TO}}-\omega_{\mathrm{LO}}$. Polarization spectroscopy experiments as outlined above gave respective values $\omega_{\mathrm{LO}}$ and $\omega_{\text {TO }}$ for these interparticle spacings. A plot of the position of $\omega_{\mathrm{LO}}$ and $\omega_{\mathrm{TO}}$ versus the interparticle spacing is shown in Fig. 3. As can be seen, the frequency difference $\Delta \omega$ of the $\mathbf{k}=0$ modes is strongly dependent on the interparticle spacing, and drops below our experimental accuracy for an interparticle distance of $5 R$ (the extinction peaks practically coincide $\left[\omega_{\mathrm{LO}}=\omega_{\mathrm{TO}}=\omega_{0}\right]$. The value of $\omega_{0}$ is deduced from the point of convergence of the extinction peaks. A fit of both curves for the longitudinal and transverse modes shows a $d^{-3}$ dependence of the position of the extinction peaks, which is consistent with the coupled dipole model for plasmon waveguides. ${ }^{12}$ Also, the peak shifts of the longitudinal collective modes with respect to $\omega_{0}$ are larger than the shifts of the transverse collective modes as predicted by our dipole theory. For plasmon waveguides with individual nanoparticles spaced $75 \mathrm{~nm}$ apart, the ratio of the peak shifts is $2.3: 1$, close to the predicted value of $2: 1$. The difference is attributed to the small inherent optical anisotropy of individual particles outlined above. The strong dependence of the peak shifts on the interparticle spacing and the consistency with the dipole model of plasmon waveguides shows that the observed effects are indeed the result of near-field interactions between nanoparticles in plasmon waveguides. The good agreement of the experimentally probed $\mathbf{k}=0$ points of the dispersion relation with predictions from the dipole model suggests that the model is indeed valid and that plasmon waveguides should allow for energy transport of electromagnetic energy when a locally excited in a mode with a finite wavevector $\mathbf{k} .^{12}$

To determine the maximum group velocity of information transport in plasmon waveguides, the full dispersion relation for nonzero wave vectors $\mathbf{k}$ has to be known. This can be determined using the point-dipole model ${ }^{12}$ and the experimentally obtained frequencies $\omega_{\mathrm{LO}}$ and $\omega_{\mathrm{TO}}$. We calculated the most efficient resonance frequency for transport to be $\omega_{0}=3.19 \times 10^{15} \mathrm{rad} / \mathrm{s}$ and a corresponding maximum group velocity $v_{g}=4.0 \times 10^{6} \mathrm{~m} / \mathrm{s}$ for the longitudinal mode for plasmon waveguides with an interparticle distance of $75 \mathrm{~nm}$. The bandwidth for this mode which puts an upper limit to the rate of information transfer that can be achieved was calculated to be $1.4 \times 10^{14} \mathrm{rad} / \mathrm{s}$. This is in good agreement with dipole-model calculations as outlined in Ref. 12.

In conclusion, we have determined the presence of coupled modes in plasmon waveguides consisting of metal nanoparticle chain arrays. We have shown that far-field spectroscopy can be used to probe the bandwidth of the dispersion relation, and thus forms an important tool to characterize the guiding properties of plasmon waveguides. By exciting collective modes around $\mathbf{k}=0$, we found an extinction peak splitting $\Delta \omega$ of approximately $1.0 \times 10^{14} \mathrm{rad} / \mathrm{s}$ between longitudinal and transverse excitations of plasmon waveguides consisting of $\mathrm{Au}$ nanoparticles spaced $75 \mathrm{~nm}$ apart. The resonance frequency corresponding to the maximum group velocity was calculated to be $\omega_{0}=3.19$ $\times 10^{15} \mathrm{rad} / \mathrm{s}$. Local excitation of plasmon waveguides at this frequency should allow for an optical information transfer below the diffraction limit with group velocities of about $v_{g}=4.0 \times 10^{6} \mathrm{~m} / \mathrm{s}$. Plasmon waveguides represent a means of electromagnetic energy localization and transport at spatial dimensions that are a fraction of the free-space wavelength and may enable fabrication of integrated nanoscale optical components.

We would like to thank Richard Muller and Paul Maker of the Jet Propulsion Laboratory in Pasadena for expert technical assistance. This work was sponsored by the Center for Science and Engineering of Materials at Caltech and the National Science Foundation Award ECS0103543.
*Email address: haa@its.caltech.edu

${ }^{1}$ U. Kreibig and M. Vollmer, Optical Properties of Metal Clusters (Springer-Verlag, Berlin, 1994).

${ }^{2}$ C. Bohren and D. Huffman, Absorption and Scattering of Light by Small Particles (Wiley, New York, 1983).

${ }^{3}$ T. Klar, M. Perner, S. Grosse, G. von Plessen, W. Spirkl, and J. Feldmann, Phys. Rev. Lett. 80, 4249 (1998).

${ }^{4}$ B. Lamprecht, G. Schider, R. T. Lechner, H. Ditlbacher, J. R. Krenn, A. Leitner, and F. R. Aussenegg, Phys. Rev. Lett. 84,
4721 (2000).

${ }^{5}$ S. Linden, J. Kuhl, and H. Giessen, Phys. Rev. Lett. 86, 4688 (2001).

${ }^{6}$ M. Salerno, N. Felidj, J. R. Krenn, A. Leitner, F. R. Aussenegg, and J. C. Weeber, Phys. Rev. B 63, 165422 (2001).

${ }^{7}$ M. Quinten, A. Leitner, J. R. Krenn, and F. R. Aussenegg, Opt. Lett. 23, 1331 (1998).

${ }^{8}$ J. R. Krenn, A. Dereux, J. C. Weeber, E. Bourillot, Y. Lacroute, J. P. Goudonnet, G. Schider, W. Gotschy, A. Leitner, F. R. Auss- 
enegg, and C. Girard, Phys. Rev. Lett. 82, 2590 (1999).

${ }^{9}$ R. M. Dickson and L. A. Lyon, J. Phys. Chem. B 104, 6095 (2000).

${ }^{10}$ S. A. Maier, M. L. Brongersma, and H. A. Atwater, Appl. Phys. Lett. 78, 16 (2001).

${ }^{11}$ S. A. Maier, M. L. Brongersma, and H. A. Atwater, Mater. Sci. Eng. C 19, 291 (2002).

${ }^{12}$ M. L. Brongersma, J. W. Hartman, and H. A. Atwater, Phys. Rev. B 62, R16356 (2000).
${ }^{13}$ S. A. Maier, M. L. Brongersma, P. G. Kik, Scheffer Meltzer, Ari A. G. Requicha, and H. A. Atwater, Adv. Mater. 13, 1501 (2001).

${ }^{14}$ G. Schider, J. R. Krenn, W. Gotschy, B. Lamprecht, H. Ditlbacher, A. Leitner, and F. R. Aussenegg, J. Appl. Phys. 90, 3825 (2001).

${ }^{15}$ J. M. Gerardy and M. Ausloos, Phys. Rev. B 25, 4204 (1982).

${ }^{16}$ M. Quinten and U. Kreibig, Appl. Opt. 32, 6173 (1993). 